\title{
A Retrospective Study of the Variability in Etiological Agents of Urinary Tract Infections among Patients in Windhoek-Namibia
}

\author{
Niitembu Janyenga Veronika Jatileni' ${ }^{1}$, Innocent Maposa ${ }^{2}$, Rooyen Tinago Mavenyengwa1,3* \\ ${ }^{1}$ Department of Health Sciences, School of Health and Applied Sciences, Polytechnic of Namibia, Windhoek, \\ Namibia \\ ${ }^{2}$ Department of Mathematics and Statistics, School of Health and Applied Sciences, Polytechnic of Namibia, \\ Windhoek, Namibia \\ ${ }^{3}$ Department of Medical Microbiology, University of Zimbabwe College of Health Sciences, Harare, Zimbabwe \\ Email: "rmavenyengwa@yahoo.com
}

Received 7 September 2015; accepted 24 November 2015; published 27 November 2015

Copyright (C) 2015 by authors and Scientific Research Publishing Inc.

This work is licensed under the Creative Commons Attribution International License (CC BY).

http://creativecommons.org/licenses/by/4.0/

(c) (i) Open Access

\begin{abstract}
Urinary tract infections (UTIs) are one of the most common infections that affect patients of both genders of all age groups. The common bacteria causing UTIs have not yet been identified in Namibia. Due to empirical treatment in the country, antibiotic resistance might be on the rise. The objective of the study was to identify the organisms that frequently caused UTIs, and the antibiotic sensitivity patterns of the bacteria isolated. A retrospective analysis was performed on 20,438 urine results submitted to the Namibia Institute of pathology (NIP), the public health laboratory in the country from January 2012 to December 2012. The raw data from NIP was compiled using Microsoft Excel. It was then imported to the IBM SPSS 22 statistical program for further analysis. The results showed that there were $3865(18.9 \%)$ UTI cases due to Escherichia coli making it the most prevalent organism isolated, followed by Proteus mirabilis 758 (3.7\%), Enterococcus faecalis 706 (3.5\%) and Klebsiella pneumoniae 640 (3.1\%). Female patients were more affected by UTIs than males. The eleven most common causes of UTIs in this study were mostly isolated from females. The most common cause of urinary tract infections in males was Extended Spectrum Beta Lactamase (ESBL) Klebsiella pneumoniae. The drugs to which these common organisms were resistant to were amoxicillin and cotrimoxazole (SXT). Cefapime, ofloxacin and piptaze were the most effective antibiotics in this study. There were 6 cases of UTIs due to Mycobacterium tuberculosis and 10 cases due to Schistosoma haematobium. The most common UTI etiology in Windhoek was Escherichia coli. Most of the isolates were resistant to at least one antibiotic, with ESBL organisms having
\end{abstract}

\footnotetext{
*Corresponding author.
} 
resistance to more than ten antibiotics.

Keywords

Urinary Tract Infection, Bacteria, Antibiotic Resistance, Namibia

\section{Introduction}

Urinary tract infections (UTIs) are the result of the intrusion of the urinary tract by microbial agents. They are rated as some of the most common infections in the world. Bacteria are mostly responsible for most of the UTIs worldwide [1]. Urinary tract infections affect both male and female patients of all age groups [2]. The migration of microbes from the bowel flora to the urethra is the most common route of infection in females [3]. Microscopy, culture and urine chemistry are the most common laboratory diagnostic tools used to detect causative agents of UTIs and their antibiotic sensitivity patterns. Due to emerging resistance, antibiotic sensitivity patterns have become important in informing appropriate treatment [2].

Urinary tract infections mostly occur in women than in men due to their anatomical differences. A shorter and wider urethra in women than in men makes it easier for bacteria to make their way to the urinary tract [4]. Women also have more moisture around their urethra than men, causing microbes to be trapped [5]. Wiping from back to front enhances the spread of organisms to the urinary tract. The common symptoms of UTIs include: dysuria, pyuria, nocturia, increased frequency of micturition, urgency to urinate, cloudy or dark urine or hematuria, lower abdominal pain and back discomforts, nausea and spewing, loss of appetite, fever, fatigue and foul smelling urine [4] [6].

The risk factors associated with acquiring UTIs include: diabetes, immune suppression, hypertension, allergies, increased sexual activity, catheterization, use of diaphragms, birth control pills and spermicidal agents, age and gender, delays in micturition and abuse of antibiotics [5] [7]-[9]. In most developing countries, especially in Africa, malnutrition, poor hygiene and low economic status are part of the risk factors of UTIs [3] [8]. The ABO blood group has also been regarded as a risk factor for UTIs [5].

There are a number of drugs universally used against microbes causing UTIs such as tobramycin, kanamycin, gentamycin, ciprofloxacin, amikacin, and cotrimoxazole where some resistance has been detected [7] [8] [10]. Studies have shown the resistance of $E$. coli to a variety of drugs such as sulfamethoxazole, ampicillin, cephalothin, ciproflaxin, amoxilin, augmentin, gentamycin and other medications [3] [8] [10].

Gram negative organisms are mostly isolated particularly E. coli and K. pneumoniae. The common gram positive isolates are $S$. aurues and S. epidermidis [5] [9]-[11]. Past studies have shown that the common etiological agents of urinary tract infections include E. coli causing up to 80\% of the infections [8] [10]. According to most international studies; E. coli, Klebsiella sp., Enterococcus sp., Proteus sp., Pseudomonas aeruginosa and Candida sp. are the most commonly isolated organisms [5] [9]-[12].

In Africa, women are the frequent victims of UTIs and E. coli is also said to be the most common agent isolated [4] [13]. However, a study done in Benin in Midwestern Nigeria proved otherwise. In this study, males were more affected by UTIs than females and Alcaligenes species were the most common isolates from patients, followed by K. aerogenes, then E. coli [3]. In another study, Klebsiella species were the leading uropathogens in both sexes of out-patients, followed by E. coli, S. aureus and then C. albicans [14]. A study done in Benin City in Nigeria showed that $S$. aureus was the most common organism isolated from both genders [15]. Emergence of antibiotic resistance in UTI causing organisms is a serious public health problem particularly in the developing world [3] [12].

The study was aimed at determining the common etiological agents of urinary tract infections, their antibiotic sensitivity patterns, and the most affected gender by the UTIs in patients referred to Namibia Institute of Pathology (NIP) in Windhoek throughout 2012.

\section{Methodology}

\subsection{Study Design}

This research was a descriptive retrospective study done at the Namibia Institute of Pathology (NIP), located at 
Windhoek Central Hospital, Namibia. This laboratory caters for all the laboratory tests requested from the Central Hospital and clinics around the capital city. The samples came from both outpatients and those admitted in wards including Intensive Care Unit and oncology. The selection criteria for the data used in the study was urine specimens that were collected for analysis from patients who came to the health institution presenting with signs and symptoms of a possible UTI and were screened using the urine test strip and microscopy. The data collected from patients attended to by clinicians from January to December 2012 at NIP in Windhoek was used. The data on comorbidities was not included as it was not available for some patients.

The population size of the study was 20,438 patients, comprising all the urine records that met the described selection criteria at NIP that year. The sampling method was purposive focusing on patient files who submitted urine specimens for urinary analysis in 2012 where data on infecting organisms and antibiotic sensitivity test results were available. These urine records excluded pregnancy tests, and any other investigations that did not relate to UTIs.

\subsection{Data Collection and Analysis}

A soft copy of the urine records submitted to the laboratory suspected of having a UTI in 2012, from NIP was obtained. The data was captured and stored using Microsoft Excel. The data collected was for 20,438 patients and it included the gender, date of birth, location (ward patients or outpatients), specimen date, names of the organisms isolated and their codes. It also included the antibiotic sensitivity pattern of each organism isolated. Data on other clinical comorbidities was missing for the majority of patients and hence was not included.

The data set, which excluded the patients' names was then imported to the IBM SPSS 22 statistical program for further analysis. Tables were used to summarize the proportions of the different organisms causing UTIs. The frequency of infection by different microbes in male and female patients was also analyzed. Bar charts were used to compare the antibiotic sensitivity patterns of the common isolates and the gender that is mostly affected, most affected age group and the top ten common microbes that cause UTIs in males and females.

\subsection{Ethical Considerations}

Written permission to conduct the study was sought and granted by the Namibia Institute of Pathology Ethics Committee. Permission was also sought and granted from the Ministry of Health and Social Services Ethics Committee. The patients’ names were removed from the raw data to ensure confidentiality.

\section{Results}

\subsection{The Common Agents of UTIs}

From a total of 20,438 patients, 12,482 (61.1\%) were females and 7956 (38.9\%) were males. Out of this total, 12,211 (59.7\%) had specimens from which growth was detected. Out of these 119 different species of microorganisms where isolated and they varied from Gram positive to Gram negative bacteria, Mycobacteria, fungi, parasites, mixed species and contaminants were also detected.

The 11 most common type of organisms isolated led by Escherichia coli 3865 (18.9\%), are shown in Table 1. Patients who were infected with yeasts which was not Candida albicans were 492 (2.4\%), ESBL's Esherichia coli 487 (2.4\%), Candida albicans 413 (2.0\%), Serratiaodorifera 353 (1.7\%), Klebsiellaoxytoca 299 (1.5\%) and Enterococcus species 290 (1.4\%) as indicated in Table 1.

Female patients were more affected by UTIs than males; with $65.5 \%$ of females having a positive growth compared to $50.0 \%$ in males. The eleven most common causes of UTIs in this study were isolated from females, with the minimum of $57.9 \%$ and the maximum of $81.1 \%$ as shown in Table 2. The common causes of urinary tract infections in males were ESBL's Klebsiella pneumoniae 74 (53.2\%), Pseudomonas aeruginosa 55 (61, 8\%) and Staphylococcus haemolyticus73 (61.9\%) as shown in Table 3. The age group mostly affected by UTI infection was the 20 - 29 in both males and females with $24.9 \%$ as shown in Figure 1.

\subsection{General Performance of the Antibiotics}

Antibiotic sensitivity testing was done using 26 types of antibiotics. However not all antibiotics where used on the same organisms. Table 4 shows a list of antibiotics and their general sensitivity patterns of all the isolates 
they were tested against. The antibiotics which had highest resistance were amoxicillin followed by cotrimoxazole. Only 5/26 drugs had resistance to organisms which was above $50 \%$. The drugs imipenem and amikacin showed the lowest resistance among the isolatedorhanisms.

Table 1. Common organisms isolated.

\begin{tabular}{ccc}
\hline Name of organism & Frequency & $\mathbf{( \% )}$ \\
\hline Escherichia coli & 3865 & 18.9 \\
Proteus mirabilis & 758 & 3.7 \\
Enterococcus faecalis & 706 & 3.5 \\
Klebsiella pneumoniae & 640 & 3.1 \\
Staphylococci epidermidis & 569 & 2.8 \\
Yeast but not Candida albicans & 492 & 2.4 \\
ESBL's Escherichia coli & 487 & 2.4 \\
Candida albicans & 413 & 2.0 \\
Serratia odorifera & 353 & 1.7 \\
Klebsiella oxytoca & 299 & 1.5 \\
Enterococcus species & 290 & 1.4 \\
Others & 11,566 & 56.6 \\
\hline Total & $\mathbf{2 0 , 4 3 8}$ & $\mathbf{1 0 0 . 0}$ \\
\hline
\end{tabular}

Table 2. Frequency of common isolated organisms in females and males.

\begin{tabular}{|c|c|c|c|c|}
\hline \multirow{2}{*}{ Organism's name } & & \multicolumn{2}{|c|}{ Sex } & \multirow{2}{*}{ Total } \\
\hline & & Female & Male & \\
\hline \multirow{2}{*}{ Escherichia coli } & No. & 2759 & 1106 & 3865 \\
\hline & $\%$ & 71.4 & 28.6 & 100.0 \\
\hline \multirow{2}{*}{ Proteus mirabilis } & No. & 535 & 223 & 758 \\
\hline & $\%$ & 70.6 & 29.4 & 100.0 \\
\hline \multirow{2}{*}{ Enterococcus faecalis } & No. & 477 & 229 & 706 \\
\hline & $\%$ & 67.6 & 32.4 & 100.0 \\
\hline \multirow{2}{*}{ Klebsiella pneumoniae } & No. & 424 & 216 & 640 \\
\hline & $\%$ & 66.3 & 33.8 & 100.0 \\
\hline \multirow{2}{*}{ Staphylococci epidermidis } & No. & 381 & 188 & 569 \\
\hline & $\%$ & 67.0 & 33.0 & 100.0 \\
\hline \multirow{2}{*}{ Yeast but not Candida albicans } & No. & 376 & 116 & 492 \\
\hline & $\%$ & 76.4 & 23.6 & 100.0 \\
\hline \multirow{2}{*}{ ESBL's Escherichia coli } & No. & 309 & 178 & 487 \\
\hline & $\%$ & 63.4 & 36.6 & 100.0 \\
\hline \multirow{2}{*}{ Candida albicans } & No. & 335 & 78 & 413 \\
\hline & $\%$ & 81.1 & 18.9 & 100.0 \\
\hline \multirow{2}{*}{ Serratia odorifera } & No. & 240 & 113 & 353 \\
\hline & $\%$ & 68.0 & 32.0 & 100.0 \\
\hline \multirow{2}{*}{ Klebsiella oxytoca } & No. & 181 & 118 & 299 \\
\hline & $\%$ & 60.5 & 39.5 & 100.0 \\
\hline \multirow{2}{*}{ Enterococcus species } & No. & 168 & 122 & 290 \\
\hline & $\%$ & 57.9 & 42.1 & 100.0 \\
\hline
\end{tabular}


Table 3. Common types of bacteria isolated from male patients.

\begin{tabular}{ccccc} 
Isolate's name & & \multicolumn{2}{c}{ Sex } & \multirow{2}{*}{ Total } \\
\cline { 3 - 4 } & & Female & Male & \\
\hline ESBL's Klebsiella pneumoniae & No. & 36 & 74 & 139 \\
Pseudomonas aeruginosa & $\%$ & 46.8 & 53.2 & 100.0 \\
& No. & 34 & 55 & 89 \\
Staphylococcus haemolyticus & $\%$ & 38.2 & 61.8 & 100.0 \\
& No. & 45 & 73 & 118 \\
& $\%$ & 38.1 & 61.9 & 100.0 \\
\hline
\end{tabular}

Table 4. The general performance of the antibiotics.

\begin{tabular}{ccccc}
\hline Antibiotic & No. organisms tested & \% sensitivity & \% resistance & \% intermediate \\
\hline Amikacin & 6501 & 92.0 & 3.6 & 4.5 \\
Amoxicillin & 8805 & 24.9 & 74.8 & 0.3 \\
Augmentin & 7655 & 67.3 & 25.5 & 7.2 \\
Ceftazadine & 783 & 36.0 & 19.7 & 44.3 \\
Chlorampenicol & 4588 & 73.1 & 24.1 & 1.7 \\
Cefuroxime & 8780 & 75.7 & 22.6 & 1.7 \\
Cefapime & 4937 & 66.7 & 31.2 & 2.1 \\
CHL & 27 & 85.2 & 14.8 & 0 \\
Ciprofloxacin & 7329 & 77.9 & 21.3 & 0.8 \\
Clindamycin & 82 & 63.4 & 34.1 & 2.4 \\
CLO & 879 & 44.9 & 56.1 & 0 \\
Erythromycin & 158 & 59.5 & 38.6 & 1.9 \\
Flucidine & 122 & 82.0 & 12.3 & 5.7 \\
Gentamycin & 8255 & 78.0 & 20.8 & 0.9 \\
Nalidixic acid & 7817 & 66.5 & 32.9 & 0.6 \\
Nitrofurantoin & 9923 & 75.5 & 17.4 & 7.2 \\
Norfloxacin & 84 & 92.9 & 7.1 & 0 \\
Oflaxiccin & 4939 & 84.1 & 15.4 & 0.5 \\
Oxacillin & 1240 & 39.2 & 59.9 & 0.9 \\
Penicillin & 2345 & 61.9 & 37.2 & 0.9 \\
Piperacillin & 47 & 57.4 & 40.4 & 2.1 \\
Piptaze & 2301 & 41.8 & 18.6 & 9.6 \\
Tetracycline & 175 & 97.0 & 0.9 \\
Cotrimoxazole & 9414 & 51.1 & 0.4 \\
Vancomycin & 1645 & & 72.8 & \\
Imipenem & 2223 & 5.0 & 2.4 \\
\hline
\end{tabular}

\subsection{Antibiotic Sensitivity Patterns of the Common Isolates}

Figure 2 indicates the number of antibiotics to which the common isolate are sensitive, resistant and intermediate.

Escherichia coli's sensitivity to antibiotics ranged from $63.8 \%$ for cefapime to $100 \%$ for CHL, norfloxacin penicillin, and vancomycin. It was resistant to amoxicillin (78.7\%), oxacillin (75.0\%) piperacillin (100\%) and cotrimoxazole (78.8\%). Proteus mirabilis's sensitivity to antibiotics ranged from $83.6 \%$ for cefapime to $100 \%$ 


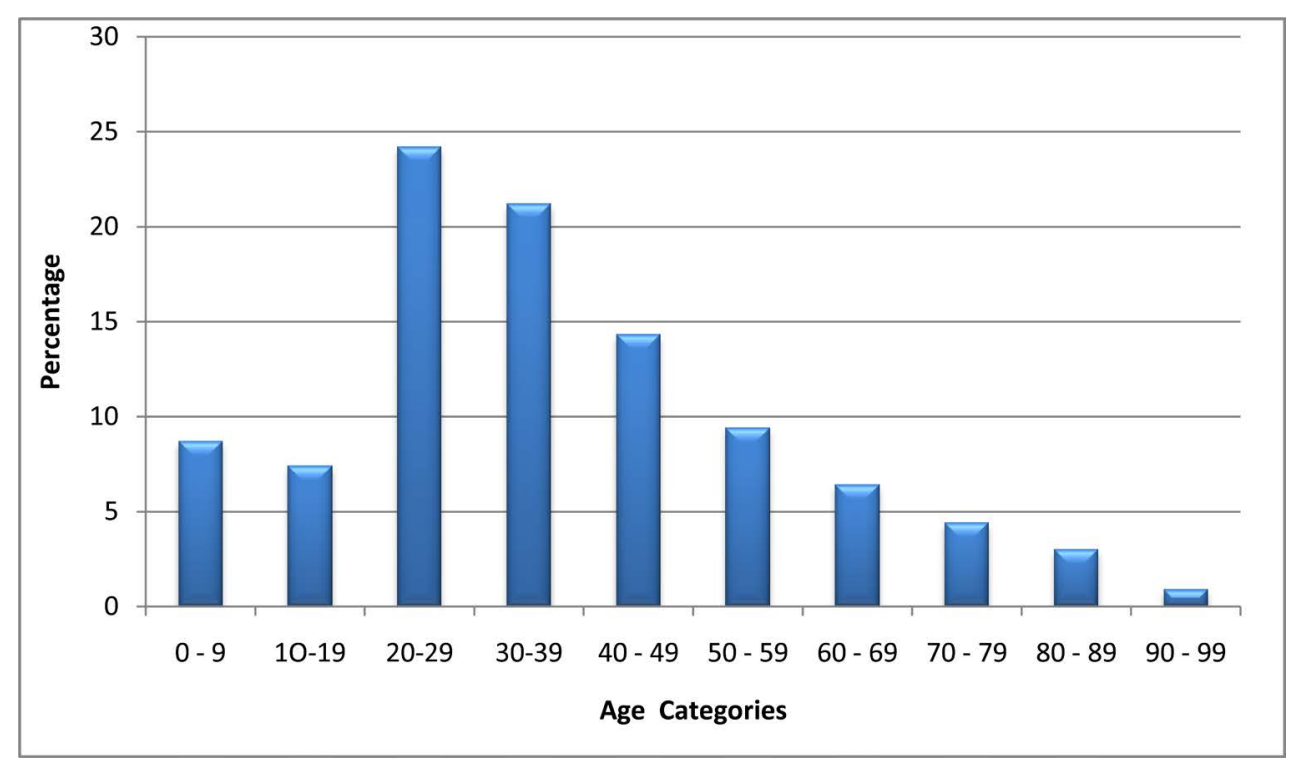

Figure 1. Proportion of patients of different age groups affected by UTIs.

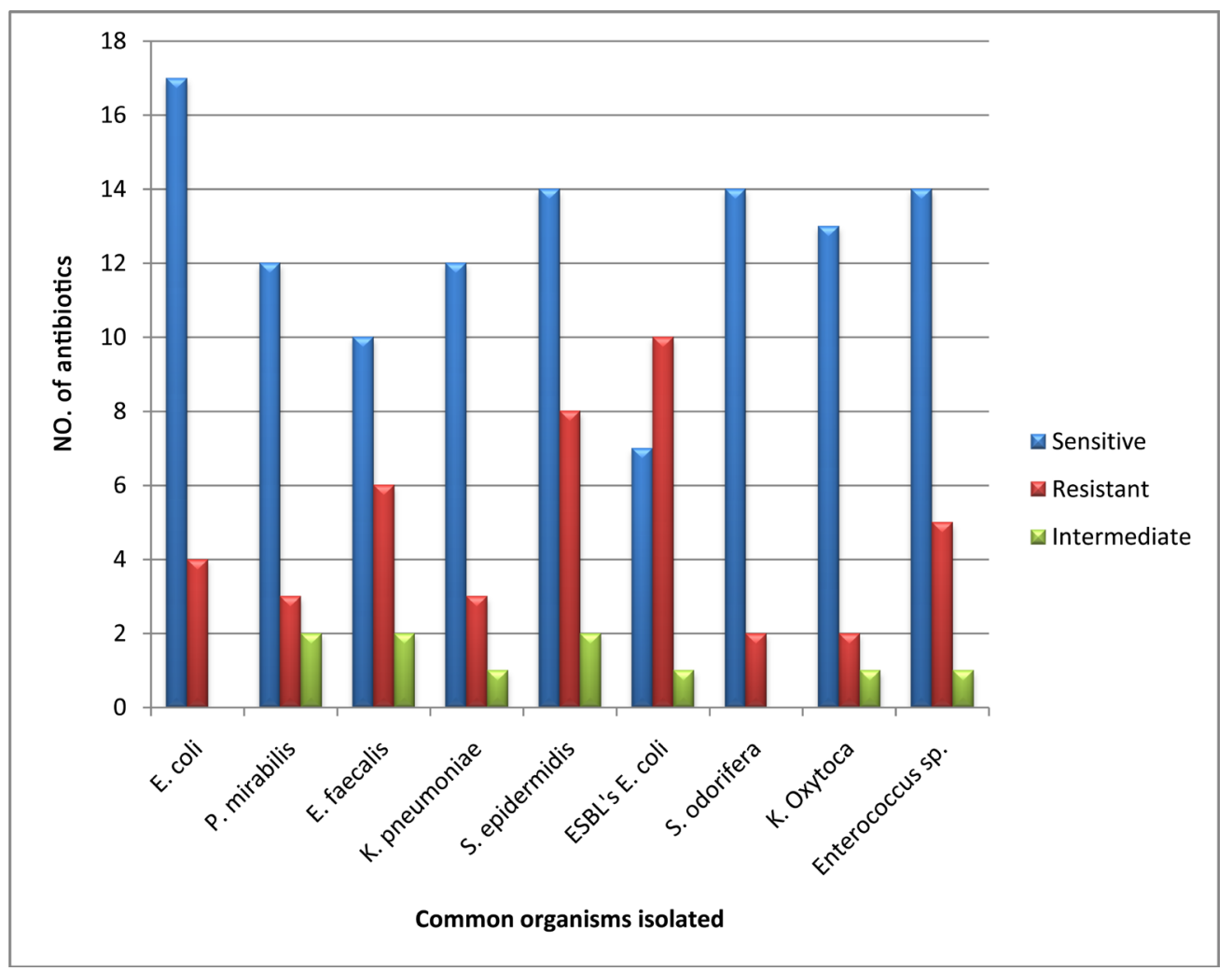

Figure 2. Antibiotics which were sensitive, resistant and intermediate resistant to the common isolates.

for piperacillin. It was resistant to amoxicillin (55.3\%), nitrofurantoin (81.7\%), cotrimoxazole (55.5\%); and intermediate to ceftazadine (66.6\%) and norfloxacin (50.0\%).

The sensitivity of Enterococcus faecalis ranged from $69.4 \%$ ofloxiccin to $99.3 \%$ for vancomycin. It was resistant to clindamycin (64.7\%), CLO (100.0\%), erythromycin (57.1\%), gentamycin (66.7\%), oxacillin (76.3\%), cotrimoxazole (97.8\%); and intermediate to chloramphenicol (50.0\%) and tetracycline (55.5\%). All Klebsiellapneumoniae isolates were sensitive to norfloxacin (100.0\%). However resistance of this organism to amoxicil- 
lin was 97.0\%, piperacillin (100.0\%), cotrimoxazole (53.5\%); and intermediate to nitrofurantoin (54.2\%).

The sensitivity of Staphylococcus epidermidis ranged from $57.1 \%$ piptaze to $100.0 \%$ amikacinand norfloxaccin. This organism had antibiotic resistance that ranged from penicillin (50.4\%) to amoxicillin (73.3\%), 100.0\% to ceftazadine and piperacillin. There was intermediate resistance to gentamycin (53.9\%) and tetracycline (50.0\%). Serratiaodorifera was found to be $100 \%$ sensitive to CHL (100.0\%), and norfloxaccin. However the S. odorifera isolates were resistant to amoxicillin (83.3\%) and cotrimoxazole (76.6\%).

Klebsiellaoxytoca isoaltes had the lowest sensitivity to cefapime (57.4\%) and the highest to CHL (100.0\%) and norfloxaccin. The K. oxytoca isolates were resistant to amoxicillin (86.8\%) cotrimoxazole (70.1\%), and intermediate to ceftazadine (66.7\%). All Extended spectrum beta lactamase $E$. coli were sensitive to penicillin and vancomycin. Their resistance ranged from gentamycin (51.9\%), to $100.0 \%$ for clindamycin and oxacillin $(100.0 \%)$.

Total sensitivity to antibiotics for Enterococcus species was recorded for cefuroxime gentamycin, nalidixic acid and tetracycline. Enterococcus species were resistant to amikacin (78.3\%), chloramphenicol (75.0\%), CLO (100.0\%), oxacillin (78.1\%), cotrimoxazole (75.0\%); and intermediate to clindamycin (50.0\%). The drugs to which the common organisms were resistant to were amoxicillin and cotrimoxazole. Cotrimoxazole was resistant to all the common isolates, while amoxicillin was resistnt to all common organisms excluding Enterococcus faecalis and Enterococcus sp. This study also revealed that cefapime, ofloxiccin and piptaze were the most effective antibiotics for the common isolates because they were sensitive to all the common isolates. Other anti-microbial agents such as augmentin, amikacin, cefuroxime, ciproflaxicin, gentamycin, nalidixic acid, nitrofurantoin, were resisted by at least one common isolate, ESBL's E. coli.

Six cases of Mycobacterium tuberculosis were found to have caused UTIs. One case of UTIs was caused by Mycobacterium other than Mycobacterium tuberculosis. Ten cases of UTIs were caused by Schistosoma haematobium.

\section{Discussion}

The study determined the common etiological agents of UTIs among patients whose urine samples were submitted to NIP. As expected E. coli (18.9\%) was frequently encountered. However, the comparatively low percentage of $E$. coli in this study does not compare to those reported by other studies. Other studies had a higher percentage of E. coli. In some European studies for instance the percentage of E. coli ranged from 35\% to 67\% [5] [7] [9] [16], while in some African studies the percentage of E. coli ranged from 32\% to 49\% [2] [13] [14].The difference in $E$. coli percentage could be due to different population sizes and the number of microbes isolated; this study's population size was 20,438 from which 119 isolates were obtained. Other studies had a much smaller population size and the number of isolates where also not so many [3] [4] [15] [17].

E. coli's prevalence was consistent with some studies around the world [2] [8] [9] [11] [13]. However, in Africa there were changes in the prevalence of microbes responsible for UTIs with Klebsiella species, Alcaligens species and Staphylococcus species being the common isolates [3] [14] [15] [18]. This could be due to the presence of different organisms in communities from different geographical regions which can potential infect the different populations.

Other frequently isolated organisms in this study were P. mirabilis, E. faecalis, K. pneumoniae, S. epidermidis, Yeast but not C. albicans, ESBL's E. coli, C. albicans, S. odorifera, K. oxytoca and Enterococcus species. However, all this isolates were mostly isolated from females than in males. The commonly isolated organisms from males were: ESBL's K. pneumoniae, P. aeruginosa and S. haemolyticus. These findings were also isolatedby other researchers not necessarily in this particular order of frequency [9] [14] [19]. Several UTI studies have indicated that most of the time the most common organisms causing UTIs in women are not the same organisms frequently isolated in males [3] [13]-[15].

Specimens received from females were more prone to UTIs than males in the current study. Apart from one study conducted in Nigeria where males were more affected by UTIs than females [3], most studies have shown that women stand a higher chance of acquiring a urinary tract infection than man [6] [7] [9] [13] [15]. This is due to the anatomical difference in women and men. In women it is easier for microbes that are normal flora in the rectum, especially the gram negative bacteria, to migrate from the anus to the urethra [14] [20]. More moisture around the urethra in females traps the microbes enabling them to spread [3]. This also explains why Candida albicans and other yeast were frequently isolated from females than males. Fungi grow and spread well in 
moist areas. The most affected age group in this study was that of 20 - 29 in both males and females with $24.9 \%$. These findings were common in most studies and this could be because the age group is the most sexually active; which is one of the common risk factors attributed to UTIs [2] [3] [8].

Data on the antibiotic sensitivity patterns of the isolated organisms was also compiled and analyzed. The drugs to which the common organisms were resistant to were amoxicillin and cotrimoxazole; they were resistant to almost all the common isolates in this study. The high resistance of amoxicillin and cotrimoxazole in this study was similar to most studies in the world [7] [12] [17] [21]-[24]. In contrast to this, a study done in the United States of America showed that E. coli was sensitive to cotrimoxazole [25]. Other anti-microbial agents such as augmentin, amikacin, cefuroxime, ciprofloxacin, gentamycin, nalidixic acid, nitrofurantoin, were resisted by at least one common isolate, especially ESBL's E. coli. These findings partially corresponded to somestudies, which indicated them as the most effective drugs and while others indicated that they were also resisted by ESBL's E. coli [3] [4] [7] [10] [13] [17] [22]-[24].

Ten cases of UTIs were caused by Schistosoma haematobium. In another study done in Ibadan Nigeria, four cases of UTIs that were caused by Schistosoma haematobium [2].This study has indicated that the isolated bacteria are resistant to at least two antibiotics. This calls for physicians to rely on laboratory guidance before they prescribe therapy. This will minimize the chance of microorganisms developing resistant genes. There a rise in the frequency of isolation of organisms such as Serratia species and Staphylococcus haemolyticus, which according to literature are not found in large quantities in UTIs. The rise in ESBL's E. coli and ESBL's K. pneumoniae was also quite alarming [26].

The limitations of the study were that only a single laboratory was used to collect the data as other laboratories in Windhoek would have also contributed valuable information concerning urinary tract infections. Some organisms isolated had only their genus name recorded but not the species name. Furthermore not all the microbes were tested against the available antibiotics and sometimes there were only less than 5 isolates that were tested against a specific antibiotic. Available data on comorbidities could have been included and analyzed to show any relationship between UTIs and other clinical factors.

\section{Conclusion}

The common etiology of UTIs in Windhoek was Escherichia coli and women were mostly affected than men. Most of the isolates were resistant to at least one antibiotic, although ESBL's had resistance to more than ten antibiotics. The common causes of urinary tract infections in males were ESBL's Klebsiella pneumoniae. The most ineffective drugs for this study were amoxicillin cefapime, ofloxiccin and piptaz and cotrimoxazole.

\section{Acknowledgements}

The authors would like to thank the Namibia Institute of Pathology for providing access to the data used in the study.

\section{References}

[1] Janifer, J., Geethalakshmi, S., Satyavani, K. and Viswanathan, V. (2009) Prevalence of Lower Urinary Tract Infection in South India Type 2 Diabetic Subjects. Indian Journal of Nephrology, 19, 107-111. http://dx.doi.org/10.4103/0971-4065.57107

[2] Okonko, I.O., Ijandipe, L.A., Ilusanya, O.A., Donbraye-Emmanuel, O.B., Ejembi, J., Udeze, A.O., Egun O.C., Fowotade, A. and Nkang, A.O. (2009) Incidence of Urinary Tract Infection (UTI) among Pregnant Women in Ibadan, SouthWestern Nigeria. African Journal of Biotechnology, 8, 6649-6657.

[3] Otajevwo, F.D. (2013) Urinary Tract Infection among Symptomatic Outpatients Visiting a Tertiary Hospital Based in Midwestern Nigeria. Global Journal of Health Sciences, 5, 187-199. http://dx.doi.org/10.5539/gjhs.v5n2p187

[4] Koffuor, G.A., Boye, A., Siakwa, P.M., Boampong, J.N., Ephraim, R.K.D., Patrick Amoateng, P., Obodai, G. and Penu, D. (2012) Asymptomatic Urinary Tract Infections in Pregnant Women Attending Antenatal Clinic in Cape Coast, Ghana. Journal of Medical Research, 1, 74-83.

[5] Behzadi, P., Behzadi, E., Yazdanbod, H., Aghapour, R., Chshmeh, M.A. and Omran, D.S. (2010) A Survey on Urinary Tract Infections Associated with the Three Most Common Uropathogenic Bacteria. Maedica-A Journal of Clinical Medicine, 5, 111-115.

[6] Ashok, C., Kumar, G.V. and Viswanathakumar, H.M. (2013) Study of the Prevalence and Clinical Profile of Urinary 
Tract Infection in Febrile Children Aged 3 - 6 Years Who Attended Pediatric Outpatient Department in a Tertiary Care Hospital. International Journal of Health Sciences and Research, 3, 1-5.

[7] Beyene, G. and Tsegaye, W. (2011) Bacterial Uropathogens in Urinary Tract Infection and Antibiotic Susceptibility Pattern in Jimma University Specialized Hospital, Southwest Ethiopia. Ethiopian Journal of Health Sciences, 21, 141146. http://dx.doi.org/10.4314/ejhs.v21i2.69055

[8] Olandeinde, B.H., Omoregie, R., Olley, M. and Anunibe, J.A. (2011) Urinary Tract Infection in a Rural Community of Nigeria. North American Journal of Medical Sciences, 3, 75-77. http://dx.doi.org/10.4297/najms.2011.375

[9] Magliano, E., Grazioli, V., Deflorio, L., Leuci, A.I., Mattina, R., Romano, P. and Cocuzza, C.E. (2011) Gender and Age Dependant Etiology of Community Acquired Urinary Tract Infections. The Scientific World Journal, 2012, 1-6. http://dx.doi.org/10.1100/2012/349597

[10] Amin, M., Mehdinejad, M. and Pourdangehi, Z. (2009) Study of Bacteria Isolated from Urinary Tract Infections and Determination of Their Susceptibility to Antibiotics. Jundishapur Journal of Microbiology, 2, 118-123.

[11] Nicolle, L.E. (2005) Complicated Urinary Tract Infection in Adults. Canadian Journal of Infectious Diseases Medical Microbiology, 16, 349-360.

[12] Ochada, N.S., Nasiru, I.A., Thairu, Y., Okanlowan, M.B. and Abdulakeem, Y.O. (2015) Antimicrobial Susceptibility Pattern of Urinary Pathogens Isolated from Two Tertiary Hospitals in Southwestern Nigeria. African Journal of Clinical and Experimental Microbiology, 16, 12-22. http://dx.doi.org/10.4314/ajcem.v16i1.3

[13] Chedi, B.A.Z., Wannang, N.N., Halliru, M.A. and Bichi, L.A. (2009) A Seven Months Retrospective Study in Urinary Tract Infection among Patients at Aminu Kanu Teaching Hospital, Kano, Nigeria. Bayero Journal of Pure and Applied Sciences, 2, 95-95.

[14] Osazuwa, F., Mordi, R.M., Osazuwa, E., Taiwo, S.S., Alli, O.A.T., Ogbolu, D.O. and Anukam, K.C. (2010) Klebsiella Has Taken Lead among Uropathogens in University of Benin Teaching Hospital, Benin City, Nigeria: An Observation. New York science Journal, 3, 61-64.

[15] Omoregie, R., Erebor, J.D., Ahonkhai, I., Isibor, J.O. and Ogefere, H.O. (2008) Observed Changes in the Prevalence of Uropathogens in Benin City Nigeria. New Zealand Journal of Medical Laboratory Sciences, 62, 29-31.

[16] Hooton, T.M., Scholes, D., Hughes, J.P., Winter, C., Roberts, P.L., Stapleton, A.E., Stergachis, A. and Stamm, W.E. (1996) A Prospective Study of Risk Factors for Symptomatic Urinary Tract Infection in Young Women. The New England Journal of Medicine, 335, 468-474. http://dx.doi.org/10.1056/NEJM199608153350703

[17] Bours, P.H.A., Polak, R., Hoepelman, A.I.M., Delgado, E., Jarquin, A. and Matute, A.J. (2010) Increasing Resistance in Community-Acquired Urinary Tract Infections in Latin America, Five Years after the Implementation of National Therapeutic Guidelines. International Journal of Infectious Diseases, 14, 770-774. http://dx.doi.org/10.1016/j.ijid.2010.02.2264

[18] Tula, M.Y. and Iyoha, O. (2014) Distribution and Antibiotic Susceptibility Pattern of Bacterial Pathogens Causing Urinary Tract Infection in Mubi Geberal Hospital, Yola, Nigeria. British Journal of Medicine and Medical Research, 4, 3591-3602. http://dx.doi.org/10.9734/BJMMR/2014/9525

[19] Bouza, E., San Juan, R., Munoz, P., Voss, A. and Kluytmans, J. (2001) A European Perspective on Nosocomial Urinary Tract Infections II. Report on Incidence, Clinical Characteristics and Outcome (ESGNI-004 Study). European Society of Clinical Microbiology and Infectious Diseases, 7, 532-542.

[20] Harper, M. and Fowlis, G. (2007) Management of Urinary Tract Infections in Men. Trends in Urology, Gynecology and Sexual Health, 12, 30-35. http://dx.doi.org/10.1002/tre.8

[21] Orenstein, R. and Wong, E.S. (1999) Urinary Tract Infections in Adults. American Family Physician, 59, $1225-1234$.

[22] Randrianirina, F., Soares, J.L., Carod, J.F., Ratsima, E., Thonnier, V., Combe, P., Grosjean, P. and Talarmin, A. (2007) Antimicrobial Resistance among Uropathogens That Cause Community-Acquired Urinary Tract Infections in Antananarivo, Madagascar. Journal of Antimicrobial Chemotherapy, 59, 309-312. http://dx.doi.org/10.1093/jac/dkl466

[23] Bercion, R., Mossoro-Kpinde, D., Manirakiza, A. and Le Faou, A. (2009) Increasing Prevalence of Antimicrobial Resistance among Enterobacteriaceae uropathogens in Bangui, Central African Republic. Journal of Infections in Developing Countries, 3, 187-190. http://dx.doi.org/10.3855/jidc.34

[24] Mekki, A.H., Hassan, A.N. and Elsayed, D.E.M. (2010) Extended Spectrum Beta Lactamases among Multi Drug Resistant Escherichia coli and Klebsiella Species Causing Urinary Tract Infections in Khartoum. Journal of Bacteriology Research, 2, 18-21.

[25] Grover, M.L., Bracamonte, J.D., Kanodia, A.K., Edwards, F.D. and Weaver, A.M. (2009) Urinary Tract Infection in Women Over the Age of 65: Is Age Alone a Marker of Complication? Journal of the American Board of Family Medicine, 22, 266-271. http://dx.doi.org/10.3122/jabfm.2009.03.080123

[26] Shahlol, A.M., Abukhres, O.M. and Taher, I.A. (2015) Prevalence and Characterization of Extended-Spectrum $\beta$-Lactamase-Producing Enterobacteriaceae in Brack-Alshati, Fezzan, Libya. EC Microbiology, 1, 23-32. 